\title{
Local recurrence of a phyllodes tumour of the breast presenting with widespread differentiation to a telangiectatic osteosarcoma
}

\author{
J F Graadt van Roggen, H M Zonderland, K Welvaart, J L Peterse, P C W Hogendoorn
}

\begin{abstract}
Osteogenic sarcomas of the breast are extremely rare and need to be distinguished from a variety of breast lesions producing metaplastic bone. A 50 year old patient presented with a painless lump in her right breast after twice previously having undergone local excision of a phyllodes tumour at this site. Following radiological and cytological investigation, excision was advised. Histology showed focal remnants of the previously excised phyllodes tumour in continuity with areas of widespread differentiation towards a telangiectatic osteosarcoma. So far this is a unique morphological endpoint. (f Clin Pathol 1998;51:706-708)
\end{abstract}

Keywords: extraskeletal osteosarcoma; breast; phyllodes tumour

Extraskeletal osteosarcoma is defined as a malignant mesenchymal neoplasm producing neoplastic osteoid or bone and occurring

Leiden University Medical Centre,

Leiden, The

Netherlands

Department of

Pathology

J F Graadt van Roggen

P C W Hogendoorn

Department of Radiology

$\mathrm{H}$ M Zonderland

Department of

Surgery

K Welvaart

Antoni van

Leeuwenhoek Hospital, Amsterdam, The

Netherlands

Department of

Pathology

J L Peterse

Correspondence to: Dr J F Graadt van Roggen, Department of Pathology Building I, L1-Q, Leiden University Medical Centre, PO Box 9600, 2300RC Leiden, The Netherlands; email: jgraadt@

path_1.medfac.leidenuniv.nl

Accepted for publication 28 May 1998

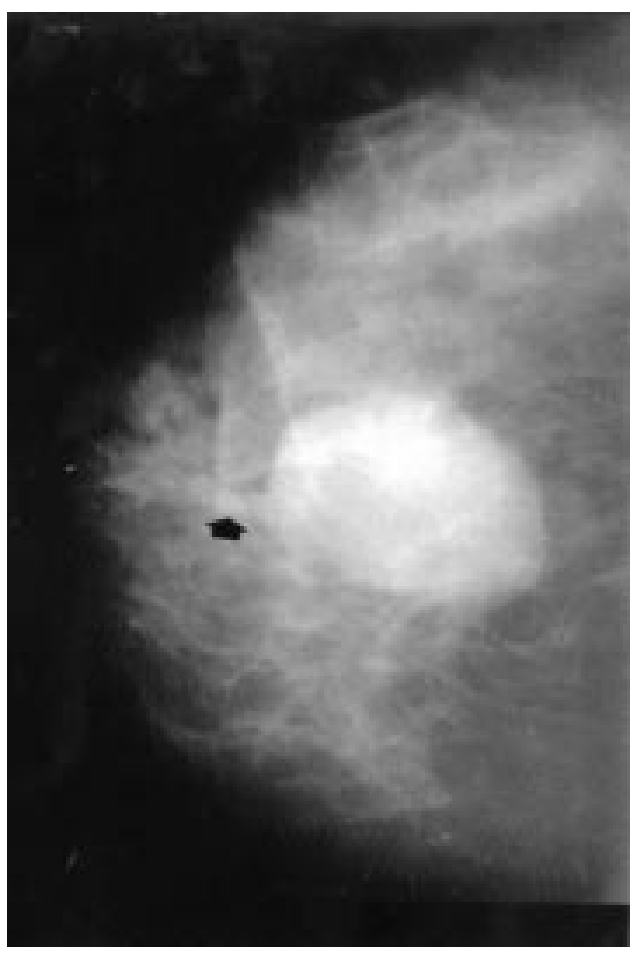

Figure 1 Mammography of the right breast at time of the latest recurrence. The fibroglandular tissue shows involution. The recurrent mass is sharply delineated without any spiculations or calcification identifiable. The fibrous tissue between the mass and nipple (black arrow) is regarded as postoperative scar tissue. within the soft tissues free from any underlying bone as determined radiographically or by surgical inspection. ${ }^{1-4}$ Reliable data documenting the incidence of extraskeletal osteosarcomas are scarce but these neoplasms appear to account for $1.25-1.65 \%$ of all soft tissue sarcomas. ${ }^{1}$

True osteogenic sarcomas of the breast are extremely rare and need to be distinguished from a variety of benign and malignant breast lesions producing metaplastic bone. ${ }^{56} \mathrm{Un}$ equivocal data on the incidence of osteosarcoma occurring in the breast are not readily available but an estimate of approximately $1 / 1000$ breast malignancies is probably not unreasonable. ${ }^{14}$ The histogenesis, as well as the relation to other breast lesions, is uncertain although various hypotheses have been debated (see the discussion). We describe a mesenchymal extraskeletal tumour showing the unequivocal morphology of a telangiectatic osteosarcoma, arising in the breast in intimate association with a previously excised phyllodes tumour.

\section{Case report}

A 50 year old white female recently presented with a five month history of an enlarging painless mass in her right breast. The patient gave no history of nipple discharge or hormone treatment and there was no family history of breast cancer. Eight years earlier a discrete lesion $(2 \times 2 \times 2 \mathrm{~cm})$ had been completely excised from the same area, entirely embedded

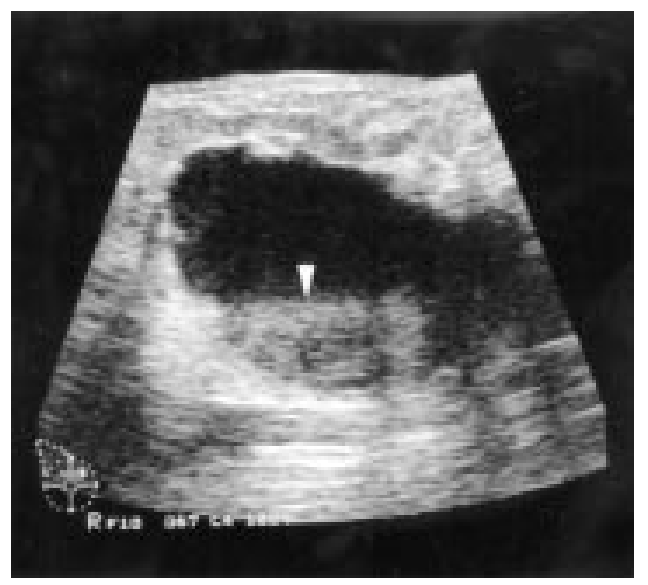

Figure 2 Sonography of the recent mass shows a complex cystic lesion with an irregular inner outline and a fluid-fluid level (arrowhead). The lower layer is echogenic, compatible with sedimented blood; the upper part shows an echo-poor aspect and seems to be more fluid. As a result of its liquid contents the mass shows enhanced sound transmission. 


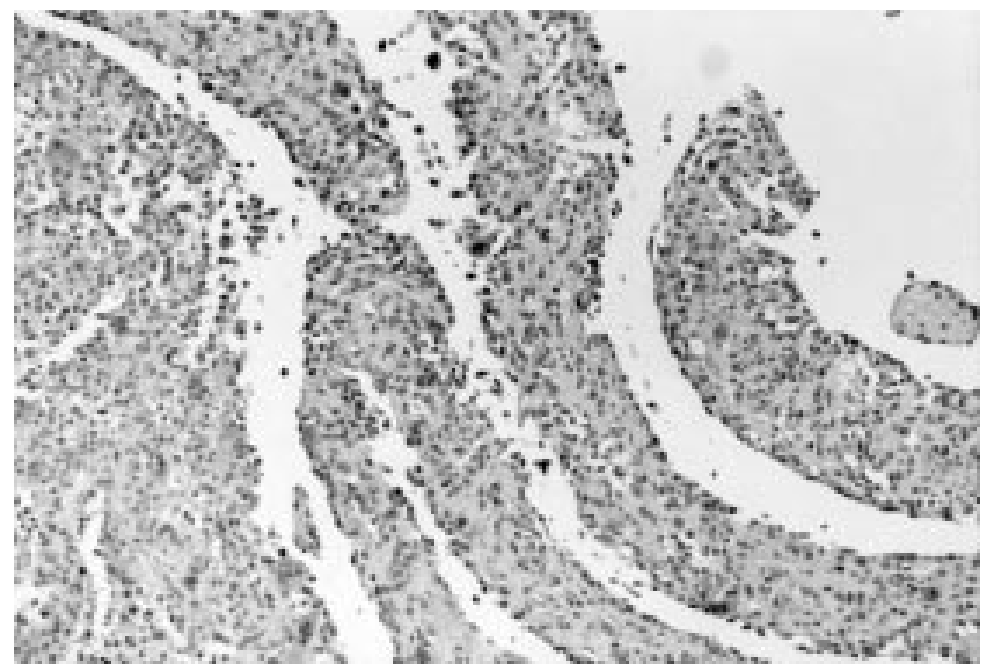

Figure 3 Low power view showing the architecture typical of a telangiectatic osteosarcoma with septa separating blood filled cystic formations. (Haematoxylin and eosin stain.)
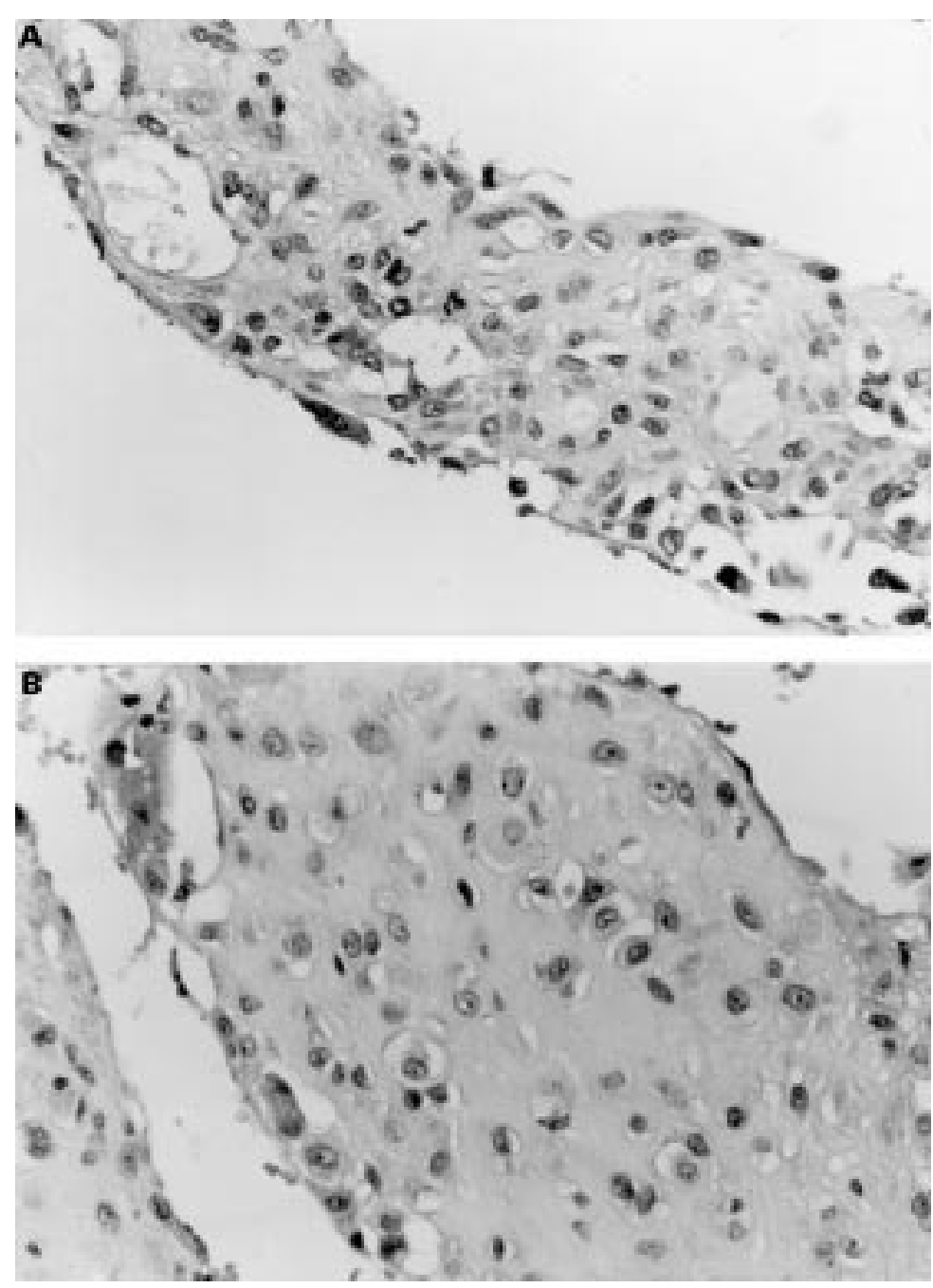

Figure 4 High power view of a septal structure within areas of telangiectatic osteosarcomatous differentiation showing irregular oriented cells with polymorphic nuclei and a mitosis (A). Subtle foci of osteoid are identifiable (B). (Haematoxylin and eosin stain.)

for histology, and originally been diagnosed as a fibroadenoma; a local recurrence $(7.5 \times 7 \times$ $6.5 \mathrm{~cm}$ ), of which just less than $50 \%$ of the lesion was included for histology and which was originally diagnosed as a giant fibro- adenoma, was completely resected four years later; no radiation treatment was given. On examination of the breast there was a deeply situated, well circumscribed, centrally situated supra-areolar mass approximately $7 \mathrm{~cm}$ in diameter. Apart from the surgical scar and associated scar tissue resulting from the previous surgery, the remainder of the breast, nipple, and areola were unremarkable. Mammography showed a $5-6 \mathrm{~cm}$ oval and well circumscribed mass. There were no radiological signs of malignancy and no calcification (fig 1). Additional sonography revealed a predominantly cystic mass with an irregular luminal outline and numerous fluid levels (fig 2). Fine needle aspiration showed dissociated, atypical, spindle shaped cells with polymorphic, hyperchromatic nuclei in a bloodstained background. The initial impression was that of a non-epithelial lesion suspicious of malignancy, and complete excision was advised.

The surgical specimen measured $8 \times 6 \times 5$ $\mathrm{cm}$. Sectioning revealed a cystic tumour, $4 \mathrm{~cm}$ in maximum diameter, with a thickened wall and an intracystic pendulous lesion, $1 \times 1 \times 1$ $\mathrm{cm}$, in continuity with the cyst wall at one site. The tumour was macroscopically free from all surgical margins, with a minimum free margin of $0.5 \mathrm{~cm}$. In addition to standard multiple sections, the entire deep margin closest to the cystic lesion was sampled for histology.

Microscopically, the cavity wall consisted of a cellular, myxoid stroma with irregular, short bundles of elongated, spindle shaped cells with mild nuclear atypia and less than one mitosis in 10 high power fields (HPF). The intracystic tumour was composed of solid, cellular areas alternating with blood filled cystic spaces separated by septa composed of disorganised, pleomorphic tumour cells with polymorphic nuclei and prominent nucleoli (figs 3 and 4). Mitoses were obvious $(\sim 1 / \mathrm{HPF})$. Between the cells infrequent patchy areas of birefringent hyaline material (osteoid) without calcification were present. No chondroid differentiation or areas with epithelial differentiation were observed. Immunohistochemically, the tumour cells were positive for vimentin and negative for keratin, CD31, CD34, S-100, and HMB-45. Based on the morphology and immunohistochemical profile, a diagnosis of telangiectatic extraskeletal osteosarcoma was made, arising as a result of the very unusual phenomenon of further malignant differentiation within a pre-existent phyllodes tumour.

In view of the histopathological diagnosis in the current resection specimen, the microscopy of the two earlier lesions was reviewed and, based on the architecture and stromal cellularity, a revised diagnosis of a phyllodes tumour followed by local recurrence (figs 5 and 6) was made. Both previous lesions had been extensively sampled, including margins, and were regarded as completely excised. No sarcomatous dedifferentiation was identified in either of the two earlier specimens.

Following definitive diagnosis the patient was started on a chemotherapy regimen with doxorubicin and cisplatin. Re-excision was not performed since the original surgical margins 


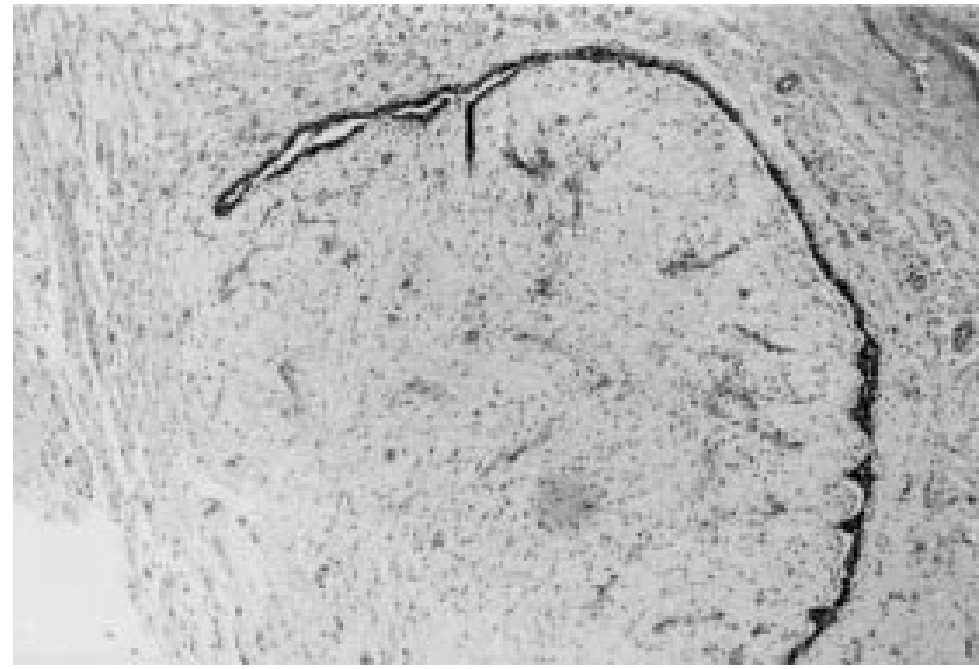

Figure 5 Low power view of the primary phyllodes tumour showing the relatively uniform cellular stroma lined by hyperplastic epithelium. (Haematoxylin and eosin stain.)

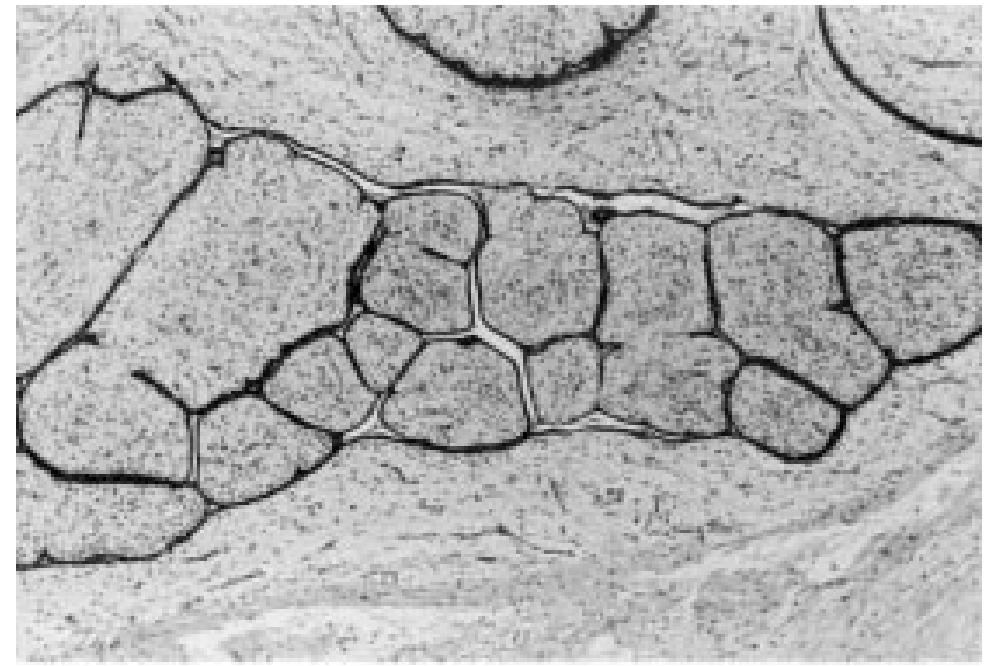

Figure 6 Low power view showing the classical club shaped architecture of the local recurrence of the phyllodes tumour. The cellular stroma and hyperplastic epithelium are evident. Areas with similar morphology were found in the second recurrence in direct continuity with areas showing unequivocal differentiation towards a telangiectatic

osteosarcoma as shown in figs 3 and 4. (Haematoxylin and eosin stain.)

were free; axillary lymph node dissection was not considered. Routine $x$ ray of the chest and a radionuclide study of the skeleton did not detect any evidence of metastatic disease. Shortly afterwards, computed tomography of the chest showed two small pulmonary nodules suspected of being metastases.

\section{Discussion}

We have documented the local recurrence of a phyllodes tumour presenting with widespread differentiation to a telangiectatic osteosarcoma, arising within the breast of a patient who had twice previously undergone local but complete excision of a phyllodes tumour. The distinction between an extraskeletal osteosarcoma and other benign and malignant bone and cartilage forming tumours may be difficult. Metaplastic bone formation may occur in fibroadenomas and phyllodes tumours. ${ }^{5}{ }^{10}$ Breast tumours with malignant bone formation are usually metaplastic carcinomas. The neoplastic epithelial component in these tumours can sometimes only be found after extensive sampling. Metaplastic bone formation may also infrequently be found in sarcomas rarely occurring in the breast such as angiosarcoma, malignant fibrous histiocytoma, pleomorphic liposarcoma, and malignant melanoma. ${ }^{1}$ The definitive diagnosis here was made on the basis of the typical histological architecture and cellular morphology, coupled with the immunohistochemical profile.

The pathogenesis of extraskeletal osteosarcomas in general is unclear. Numerous postirradiation osteosarcomas have been reported, ${ }^{1}$ while mechanical injury ${ }^{1}$ has also been proposed as a possible contributory/causative factor. In our case, no radiotherapy was given, which excludes this as a causative factor. Unlike skeletal osteosarcoma, the tumour has not been described in siblings. ${ }^{1}$

An interesting parallel would be the occurrence of true sarcomas arising from stromal elements in other benign "mixed tumours," for example mixed salivary gland tumours. These have been described but are extremely rare. ${ }^{11}$

In our case histology clearly identifies the current tumour arising in a region showing unequivocal similarity with the previously resected phyllodes tumour. A strong argument can be made supporting the controversial and rare relation between benign and "borderline" stromal tumours (fibroadenomas and phyllodes tumours, respectively) and malignant mesenchymal tumours of the breast, probably as a consequence of progressive malignant differentiation of stromal elements. Reports have appeared over the years ${ }^{78}$ in support of this concept and our present report would also serve to support this hypothesis. In our case the result is a unique variant, differentiation to a telangiectatic osteosarcoma.

1 Enzinger FM, Weiss SW, eds. Soft tissue tumours, 3rd ed. New York: CV Mosby, 1995.

2 Annani PA, Baumann RP. Osteosarcoma of the breast. Virchows Arch (Pathol Anat) 1972;357:213-18.

3 Going JJ, Lumsden AB, Anderson TJ. A classical osteogenic sarcoma of the breast: histology, immunohistochemistry and ultrastructure. Histopathology 1986;10:631-41.

4 Jernstrom P, Lindberg AL, Meland ON. Osteogenic sarcoma of the mammary gland. Am f Clin Pathol 1963;40: 521-6.

5 Smith BH, Taylor HB. The occurrence of bone and cartilage in mammary tumours. Am f Clin Pathol 1969;51:610-18.

6 Chung EB, Enzinger FM. Extraskeletal osteosarcoma. Cancer 1987;60:1132-42.

7 Rottino A, Howley CP. Osteoid sarcoma of the breast: a complication of fibroadenoma. Arch Pathol 1945;40:44-50.

8 Curran RC, Dodge OG. Sarcoma of the breast, with particular reference to its origin from fibroadenoma. $f$ Clin Pathol 1962;15:1-16.

9 Mufarrij AA, Feiner HD. Breast sarcoma with giant cells and osteoid. Am F Surg Pathol 1987;11:225-30.

10 Moffat CJC, Pinder SE, Dixon AR, et al. Phyllodes tumours of the breast: a clinicopathological review of thirty-two cases. Histopathology 1995;27:205-18.

11 Auclair PL, Langloss JM, Weiss SW, et al. Sarcomas and sarcomatoid neoplasms of the major salivary gland regions. A clinicopathologic and immunohistochemical study of 67 cases and review of the literature. Cancer 1986;58:1305-15. 